\title{
Understanding COVID-19 vaccine efficacy
}

\author{
By Marc Lipsitch ${ }^{1}$ and Natalie E. Dean ${ }^{2}$
}

${ }^{1}$ Center for Communicable Disease Dynamics, Department of Epidemiology, Harvard T.H. Chan School of Public Health, Boston, MA 02115, USA. ${ }^{2}$ Department of Biostatistics, University of Florida, Gainesville, FL 32611, USA. Email: mlipsitc@hsph.harvard.edu; nataliedean@ufl.edu

\section{Vaccine efficacy in high-risk groups and reduced viral shedding are important for protection}

The elderly and people with comorbidities are at greatest risk of severe coronavirus disease 2019 (COVID-19). A safe and effective vaccine could help to protect these groups in two distinct ways: direct protection, where high-risk groups are vaccinated to prevent disease, and indirect protection, where those in contact with high-risk individuals are vaccinated to reduce transmission. Influenza vaccine campaigns initially targeted the elderly, in an effort at direct protection, but more recently have focused on the general population, in part to enhance indirect protection. Because influenza vaccines induce weaker, shorter-lived immune responses in the elderly than in young adults, increasing indirect protection may be a more effective strategy. It is unknown whether the same is true for COVID-19 vaccines.

For COVID-19, age-structured mathematical models with realistic contact patterns are being used to explore different vaccination plans $(1,2)$, with the recognition that vaccine doses may be limited at first and so should be deployed strategically. But as supplies grow large enough to contemplate an indirect protection strategy, the recommendations of these models depend on the details of how, and how well, these vaccines work and in which groups of people. How can the evidence needed to inform strategic decisions be generated for COVID-19 vaccines?

Phase 3 vaccine trials are designed to assess individuallevel efficacy and safety. These trials typically focus on a primary endpoint of virologically confirmed, symptomatic disease to capture the direct benefit of the vaccine that forms the basis for regulatory decisions. Secondary endpoints, such as infection or viral shedding, provide supporting data, along with analyses of vaccine efficacy in subgroups. Nonetheless, unanswered questions about COVID-19 vaccine characteristics are likely to remain even after trials are completed. First, trials are typically not powered to establish subgroup-specific efficacy, yet the performance of the vaccine in high-risk groups affects the success of a direct-protection strategy. Second, can vaccines prevent infection or reduce contagiousness? This matters for achieving indirect protection. Expanding ongoing efforts or planning new studies may generate the data needed to address these questions.

For estimating subgroup-specific efficacy, randomized controlled trials can provide early estimates, yet these will have wide confidence intervals, leaving substantial uncertainty about true effects in high-risk subgroups. This uncertainty would be greater in interim analyses that are based on the number of events across the whole trial population and may be exacerbated if high-risk participants are more cautious and have lower exposure to infection, reducing their contribution to the efficacy estimates.

There are several strategies to address subgroup-specific efficacy, some of them already in place. Ensuring that highrisk adults are well represented in the trial population can be achieved by setting minimum enrollment targets for older adults and/or adults with comorbidities. Another consideration relates to the stopping rules for interim analyses in trials. Vaccine trials with early interim analyses that are planning to discontinue randomization and vaccinate placebo participants after declaring efficacy are most prone to subgroup uncertainty. To improve the precision of efficacy estimates in high-risk subgroups, regulators could insist that interim analyses be performed only after a certain number of confirmed disease cases occur in these subgroups, in addition to existing monitoring of the overall number of events in the study.

Trials that maintain blinded follow-up to assess long-term efficacy and safety may also generate more-reliable evidence on age-specific effects. For example, the World Health Organization's Solidarity Vaccines Trial will preserve placebo-controlled follow-up through month 12 or when an effective vaccine is deployed locally (3). However, depending on where the trials are being done and whether the vaccine becomes rapidly available in sufficient quantities after emergency-use authorization in the population undergoing the trial, it may become unethical and/or impractical to ask participants in some subgroups to forego access to an available vaccine. For vaccine candidates evaluated in multiple trials, such as the Oxford-AstraZeneca vaccine being studied in the United Kingdom, South Africa, Brazil, and the United States, metaanalyses can synthesize results across locations to improve precision of subgroup-specific effect estimates.

Ideally the phase 3 trials in progress will identify more than one safe, effective vaccine for regulatory approval and deployment. Postapproval studies will then take on an important role for continued assessment of vaccine effectiveness. These may include individual- or community-level randomized trials to compare different active vaccines without a control arm, as in the U.S. Department of Defense's 
individually randomized Pragmatic Assessment of Influenza Vaccine Effectiveness in the DoD (PAIVED) trial, which assesses the relative merits of three licensed influenza vaccines (NCT03734237).

Another approach to amass evidence on subgroup-specific efficacy is postapproval observational studies. This includes active surveillance of high-priority cohorts from, for example, nursing homes or assisted living facilities, as has been done for influenza. This also includes test-negative designs, which are routinely used to assess vaccine effectiveness (4). Symptomatic individuals that test negative for severe acute respiratory syndrome coronavirus 2 (SARS-CoV-2) function as controls for test-positive cases, and their vaccination status is compared, adjusting for selected confounders. Test-negative designs can be integrated into outpatient testing in the community (5) or use emergency department visits to estimate vaccine effectiveness against severe disease (6). To rapidly establish these systems, researchers can leverage ongoing influenza surveillance. Conveniently, these programs can simultaneously monitor more than one vaccine, enabling assessment of their relative merits.

A key limitation of observational studies is confounding. There may be many differences between individuals who do and do not get vaccinated, which may create noncausal correlations between vaccine status and outcomes. Although such biases can threaten any observational study of vaccine effectiveness, there are some approaches to detect such biases and reduce their magnitude $(7,8)$.

The clearest evidence of indirect protection is from a vaccine that prevents infection entirely, thereby reducing transmission. These data will be generated in efficacy trials that include infection as a secondary endpoint. This endpoint is measured by a specialized assay to distinguish an infectioninduced response from a vaccine-induced antibody response. A vaccine can provide indirect protection even if it does not fully prevent infection (see the figure). Vaccines that reduce disease severity can also reduce infectiousness by reducing viral shedding and/or symptoms that increase viral spread (e.g., coughing and sneezing). A worst-case scenario is a vaccine that reduces disease while permitting viral shedding; this could fail to reduce transmission or conceivably even increase transmission if it suppressed symptoms.

To assess a vaccine's impact on infectiousness, some phase 3 trials examine the amount or duration of viral shedding in laboratory-confirmed, symptomatic participants by home collection of saliva samples and frequent polymerase chain reaction (PCR) testing. However, this would not capture any change in viral shedding for asymptomatic participants. Moreover, serology tests detect previous infection and cannot reconstruct shedding during active infection. To measure viral load in both symptomatic and asymptomatic participants, it is necessary to conduct frequent (e.g., weekly) viral testing, irrespective of symptoms, to capture participants during their period of acute infectiousness. The OxfordAstraZeneca vaccine trial is testing participants in the United Kingdom for virus weekly regardless of symptoms, but not in other trials for which protocols have been released. Even weekly testing will not give detailed information about the effect of the vaccine on viral shedding, and the relationship between viral loads and infectiousness is unknown; nonetheless, this approach is likely to provide some evidence if viral loads are on average lower among vaccinated people. Human challenge vaccine studies, in which individuals in a randomized controlled trial are deliberately exposed to the virus, could generate high-quality data on the effect of vaccines on viral shedding (9).

Other approaches exist to directly estimate infectiousness without the need to extrapolate from viral load. Add-on household studies can supplement efficacy trials. Investigators can follow household members or other close contacts of infected participants to assess the vaccine's effect on infectiousness, as has been implemented for the respiratory disease pertussis (also called whooping cough) (10). Viral sequencing could be used within the trial to link infector-infectee pairs and better estimate indirect effects (11). Another strategy is to design cluster-randomized trials in which indirect effectiveness is a primary outcome. In influenza vaccine trials, health care workers at nursing homes were cluster-randomized to be offered vaccine or not, and the endpoints were mortality, influenza-like illness, or influenza infection in the patients they cared for (12).

Observational studies may also be helpful, but, in general, measuring indirect effects of vaccines is even harder than detecting direct effects. It is urgent, therefore, to obtain evidence on how each candidate vaccine affects infectiousness either before approval or soon after, when scarcity may justify randomized distribution of a vaccine.

Other open questions about the rapidly developed COVID19 vaccines include long-term safety (indicating the critical need for pharmacovigilance activities), the duration of vaccine protection, the efficacy of a partial vaccination series or of lower doses (13), the vaccine's level of protection against severe infection and death, efficacy by baseline serostatus, and the potential for the virus to evolve to escape vaccineinduced immunity. The answers to such questions inform the optimal use of any vaccine.

Availability of a COVID-19 vaccine will initially be limited, and so several expert committees are exploring strategic prioritization plans. Health care workers are a common first-tier group (14), which in turn preserves health care systems by protecting those who run them and need them. A next priority is to directly protect those who are at highest risk of death or hospitalization when infected: specifically, those over 65 and people with certain comorbid conditions. This strategy 
may be optimal for reducing mortality even if the vaccine is somewhat less effective in these groups (2). But if a vaccine offers little to no protection in high-risk groups yet is able to reduce infection or infectiousness in younger adults, an indirect strategy could be preferred as vaccine supplies become large enough $(1,2)$. A worst-case scenario for an effective vaccine is one that reduces disease in younger adults but provides neither direct nor indirect protection to high-risk groups, leaving the most vulnerable at risk. Knowing these vaccine characteristics is important when evaluating the relative merits of other products. Fortunately, there are many vaccine candidates in development that use a mixture of innovative and existing technologies. Although vaccines may vary in their characteristics, having reliable evidence on direct and indirect protection can help plan how to use these vaccines in a coordinated way.

\section{REFERENCES AND NOTES}

1. L. Matrajt, J. Eaton, T. Leung, E. R. Brown, medRxiv 10.1101/2020.08.14.20175257 (2020).

2. K. M. Bubar, S. M. Kissler, M. Lipsitch, S. Cobey, Y. Grad, D. B. Larremore, medRxiv 10.1101/2020.09.08.20190629 (2020).

3. P. Krause et al., Lancet (2020). 10.1016/s0140-6736(20)31821-3

4. H. Chua et al., Epidemiology 31, 43 (2020). doi:10.1097/EDE.0000000000001116 Medline

5. Centers for Disease Control and Prevention (CDC), US Flu VE Network (2020); www.cdc.gov/flu/vaccines-work/us-flu-ve-network.htm.

6. Q. Chen et al., J. Infect. Dis. 211, 1045 (2015). doi:10.1093/infdis/iiu578 Medline

7. M. Lipsitch, A. Jha, L. Simonsen, Int. J. Epidemiol. 45, 2060 (2016). Medline

8. N. E. Dean, M. E. Halloran, I. M. Longini Jr., Am. J. Epidemiol. kwaa084 (2020). 10.1093/aje/kwaa084 Medline

9. S. K. Shah et al., Science 368, 832 (2020). doi:10.1126/science.abc1076 Medline

10. M. E. Halloran, M. P. Préziosi, H. Chu, J. Am. Stat. Assoc. 98, 38 (2003). doi:10.1198/016214503388619076

11. R. Kahn et al., medRxiv 10.1101/2020.09.14.20193789 (2020).

12. W. F. Carman et al., Lancet 355, 93 (2000). doi:10.1016/S0140-6736(99)05190-9 Medline

13. S. Riley, J. T. Wu, G. M. Leung, PLOS Med. 4, e218 (2007). doi:10.1371/journal.pmed.0040218 Medline

14. National Academies of Sciences, Engineering, and Medicine, "Framework for equitable allocation of COVID-19 vaccine" (Consensus Study Report, The National Academies Press, Washington, DC, 2020).

15. M. E. Halloran, I. M. Longini Jr., C. J. Struchiner, Design and Analysis of Vaccine Studies (Springer, 2010).

\section{ACKNOWLEDGMENTS}

M.L. receives funding from cooperative agreement 1 U01CA261277 from the U.S. National Institutes of Health. N.E.D. receives funding from NIH/NIAID R01Al139761. We thank R. Venkayya and N. Grassly for helpful comments. M.L. receives honoraria and consulting fees from Merck, Affinivax, Sanofi Pasteur, and Antigen Discovery; receives research funding (institutional) from Pfizer; and provides unpaid scientific advice to Janssen, Astra-Zeneca, and Covaxx (United Biomedical).

Published online 21 October 2020

10.1126/science.abe5938 


\section{Vaccine effects}

Vaccines provide direct protection by reducing susceptibility to disease or infection. Vaccines provide indirect protection by reducing the number of people infected in a population or their infectiousness. These vaccine effects can be assessed in clinical trials by measuring the efficacy to prevent disease, to prevent infection, and to reduce infectiousness, as well as in studies to assess indirect effects of the vaccine (15).

Individually randomized vaccine efficacy trial
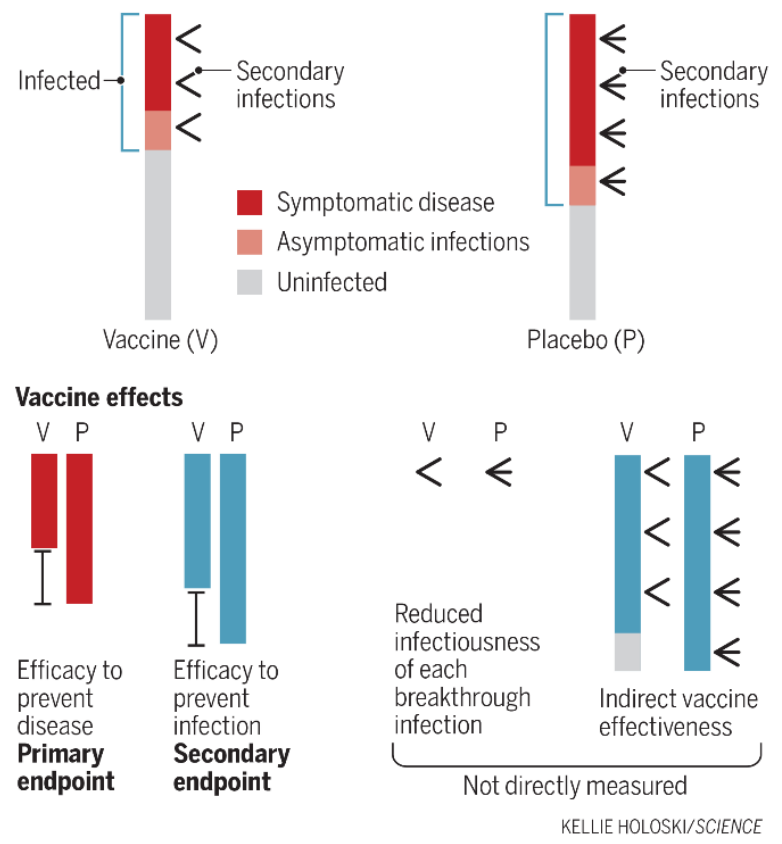